\title{
Improving the quality of care for people with giant cell arteritis
}

\author{
Authors: Chetan Mukhtyar, ${ }^{A}$ Georgina Ducker, ${ }^{B}$ Sarah Fordham, ${ }^{C}$ Sonja Mansfield-Smith ${ }^{\mathrm{D}}$ and Colin Jones ${ }^{\mathrm{E}}$
}

Giant cell arteritis (GCA) is a systemic vasculitis with numerous potential complications and societal costs. After the publication of international guidelines, we found a number of deficiencies in the local care pathway of patients suspected to have GCA. These included poor referral and management pathways, and absence of dedicated monitoring and followup. In this paper, we describe a 10-year transformation which led to our service being nominated for a national award.

A comprehensive consensus pathway saw referral numbers rise from 19 to 135 from 2012 to 2019. A consensus management pathway has meant that patients are assessed within 2 days of referral and glucocorticoids started at point of referral. All patients with suspected GCA are clerked and managed according to this agreed pathway which is available on the hospital intranet. The introduction of diagnostic ultrasonography has meant that the need for biopsies has dropped by $>80 \%$ reducing the annual cost of diagnostics by $>£ 140,000$. The introduction of a vasculitis specialist nurse has resulted in improving education, contact and speed of access to our service. The improvements in the service resulted in our service becoming a finalist in the Royal College of Physicians Excellence in Patient Care Award in 2020.

KEYWORDS: giant cell arteritis, multidisciplinary collaboration, vasculitis specialist nurse, diagnostic ultrasonography, temporal artery biopsy

DOI: 10.7861/clinmed.2021-0126

\section{Introduction}

Giant cell arteritis (GCA) is a systemic vasculitis which exclusively affects people $>50$ years of age. ${ }^{1}$ It is associated with numerous potential complications as well as societal costs. ${ }^{2}$ The first international recommendations for the management of GCA

Authors: ${ }^{A}$ consultant rheumatologist, Norfolk and Norwich University Hospital, Norwich, UK; ${ }^{\text {B }}$ specialist vasculitis and CTD Nurse, Norfolk and Norwich University Hospital, Norwich, UK;

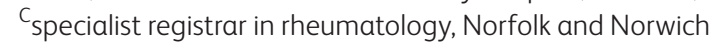
University Hospital, Norwich, UK; ${ }^{\text {D }}$ medical ophthalmology registrar, Norfolk and Norwich University Hospital, Norwich, UK; ${ }^{E}$ consultant ophthalmologist, Norfolk and Norwich University Hospital, Norwich, UK were published in $2009 .{ }^{3}$ In the UK, the annual incidence of GCA is $2.2 / 10,000$ population above the age of $50 .{ }^{4}$ Within the UK, the East of England has the highest incidence by a factor of $20 \%$ as compared to the national average. ${ }^{4}$ The Norfolk and Norwich University Hospital NHS Foundation Trust (NNUH) is the largest NHS Trust in Norfolk. Norfolk has a population of $>900,000$ which has $96.5 \%$ White ethnicity and $48.3 \%$ above the age of 50 (data from www.norfolkinsight.org.uk, accessed on 7 January 2021). We would therefore expect 100 newly diagnosed GCA in Norfolk, of which at least half should present to the Norfolk and Norwich University Hospital (NNUH).

In 2009, there were 27 cases diagnosed with GCA at NNUH. These had presented to cardiology, geriatrics, neurology, ophthalmology, rheumatology and vascular surgery. The biopsies had been done by ophthalmologists, thoracic surgeons and vascular surgeons: specimens varied in length from $0.5 \mathrm{~cm}$ to $3.5 \mathrm{~cm}$ and occasionally did not contain any vascular tissue. One case each had been biopsied in primary care and the private sector. Neither received any follow-up in the hospital. Twelve out of twenty-seven had not received any rheumatology follow-up. Of those 12 , only three had received follow-up in clinics with vasculitis expertise. None of the 27 patients had received any education about their condition or the use of long-term steroids.

The deficiencies found in the care of patients with GCA are summarised as follows (Fig 1):

> Referral: We were seeing far less GCA than we expected, and evidence suggested that some cases were solely being managed in primary care. Routine referrals could be sent to a variety of specialists and sometimes only if patients had not responded adequately to primary care treatments.

> Pharmacotherapy: There was no protocol for the use of oral or intravenous glucocorticoids and the dosage depended heavily on the specialty involved.

> Biopsy: Arranging a temporal artery biopsy was done on an ad hoc basis with a variable yield.

> Patient empowerment: Patients found it difficult to access primary care and secondary care services in a timely manner when faced with symptoms suggestive of new or relapsing disease. It was usual for prednisolone dosing to remain unchanged in the event of postponed appointments. There was no formal education about the disease or its treatments.

$>$ Follow-up and monitoring: It was the usual practice for individuals with GCA to be discharged to primary care for ongoing management without monitoring of glucocorticoid toxicity, long-term disease-related complications or relapses. 
Fig 1. The problems identified in the care of patients with GCA.
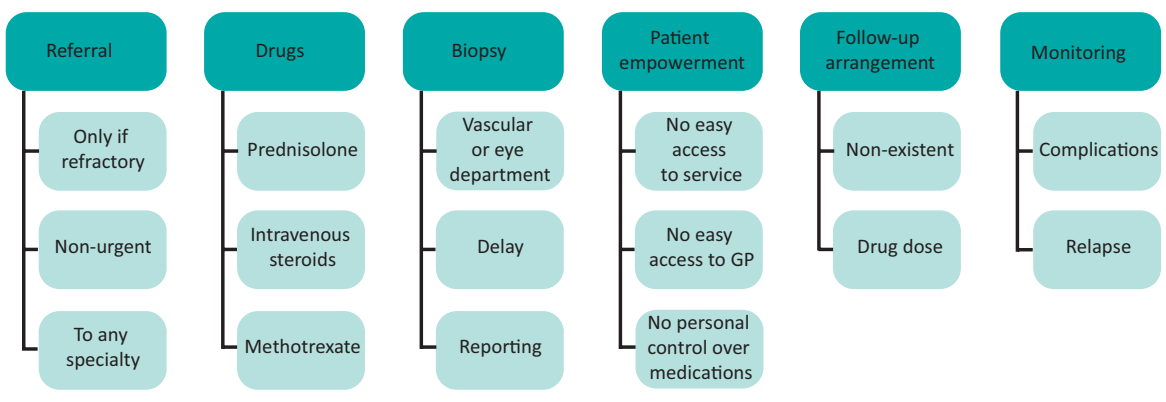

Having identified the problems, we describe our 10-year journey to improve all aspects of our service, which earned us the accolade of being finalists for the Quality Improvement Award in the Royal College of Physicians 'Excellence in Patient Care' awards in 2020 (https://bit.ly/2V8gyIK, accessed on 11 January 2021).

\section{Aims of the project}

> To create a comprehensive referral and diagnostic pathway for the timely assessment of cases with suspected GCA.

$>$ To have an agreed protocol for clerking, investigating and using glucocorticoids in the management of GCA.

> To empower patients through education about the disease, anticipated outcomes and a plan for tapering and eventual withdrawal of prednisolone.

$>$ To have an open access clinic for follow-up and monitoring of the disease, the drugs, potential complications and early diagnosis of relapses.

$>$ To improve the cost-effectiveness of the service.

\section{Interventions}

The following is the timeline of the interventions that we took to improve our service.

> December 2010: A scoping exercise to look at numbers of patients with GCA, their journeys and compare against expected numbers from published literature.

> March 2011: After a systematic literature review and mathematical modelling, the 'Norwich Regimen' for prednisolone was agreed between rheumatology and ophthalmology, presented at a national conference and published. ${ }^{5}$

> November 2011: The rheumatology and ophthalmology departments commenced negotiations for a new diagnostic pathway for GCA.

> February 2012: A new GCA clinic was formed and formally recognised on our NHS Trust's directory of services.

> March 2013: CM learnt diagnostic ultrasonography for GCA, and a service validation project was formally commenced. In the absence of formal certification of this technique, the experience was presented locally and published. ${ }^{6}$

> November 2013: First of many business meetings to offer diagnostic ultrasonography as a service to local Clinical Commissioning Groups.

> April 2014: First interdisciplinary meeting of all interested specialties to agree on a consensus Trust pathway for managing GCA.

> February 2016: The business case was finalised for offering diagnostic ultrasonography as a service to CCG.
> January 2017: Diagnostic ultrasonography (6 slots/wk) was commenced formally.

> November 2019: New Vasculitis nurse appointed, and formal education and annual review appointments commenced from February 2020.

> May 2020: Service nominated for Quality Improvement award in EPCA 2020.

\section{Study of the interventions}

The improvement in referral numbers

From the scoping exercise in December 2010, we found that of the 27 cases diagnosed with GCA, seven had presented to rheumatology and a further nine had received a follow-up appointment in a rheumatology clinic. We established a GCA clinic in February 2012 and in the first year received 19 GCA referrals. By 2019, the service received 137 referrals, of which 51 were diagnosed as having GCA. Table 1 shows the steady rise in numbers of referrals and diagnosed cases from 2012 to 2019. The spike in 2017 was a direct consequence of the establishment of the diagnostic ultrasonography service.

\section{Improving the patient journey}

An interdisciplinary group with representation from acute medicine, geriatrics, stroke medicine, neurology, ophthalmology and rheumatology first met in April 2014 to streamline the referral pathway for suspected GCA. A Trust-wide pathway was agreed which ensured that all suspected GCA with ocular symptoms would be referred to the ophthalmology team and the others to the rheumatology team. The advice and management pathway would start immediately, and formal review would happen within 24 hours for those with suspected visual involvement and 48 hours for those without. All suspected GCA cases would be clerked on a specific sheet which gave prompts for the history, examination findings and investigations. The Trust pathway and clerking sheets were made available on the Trust Intranet.

For standardisation of treatment, specific indications and contraindications were created for the use of intravenous methylprednisolone. On the basis of evidence from a systematic literature review, three statistical models were created by CM and reviewed by all members of the rheumatology department and C] for sense check and face validity. There was unanimous agreement on the use of a statistical model which was tailored to height, weight and gender of the patient. We called it the 'Norwich prednisolone regimen', and presented it locally, nationally and in a peer-reviewed publication. ${ }^{5}$ Since March 2011, all newly diagnosed patients with GCA were treated using that regimen. 
Table 1. Referrals to GCA service by year and modality of diagnosis

$\begin{array}{llllll}\text { Year } & \text { No. of referrals } & \text { Positive biopsy } & \text { Positive ultrasonography } & \text { Positive PET scan } & \text { No. of diagnosed cases } \\ 2012 & 19 & 17 & 0 & 2 & 19 \\ 2013 & 35 & 12 & 8 & 2 & 22 \\ 2014 & 52 & 10 & 13 & 2 & 25 \\ 2015 & 44 & 6 & 7 & 2 & 15 \\ 2016 & 60 & 3 & 14 & 2 & 19 \\ 2017 & 147 & 8 & 31 & 2 & 41 \\ 2018 & 135 & 8 & 37 & 5 & 50\end{array}$

The European recommendations for the management of GCA were published in 2009, and the British recommendations in 2010. 3,7 Both recommended temporal artery biopsy (TAB) for the diagnosis of GCA. However, the technique of ultrasonography had been evolving since $1995,{ }^{8}$ and we were an early adopter of the technique. CM learnt ultrasonography in March 2013 and over subsequent years validated the results against long-term outcomes. In a validation exercise involving 25 patients followed for 100 weeks, ultrasonography proved more reliable than TAB or even baseline diagnosis. ${ }^{6}$ The hospital diagnostic pathway included the use of ultrasonography for all suspected GCA followed by TAB or positron emission tomography only if the ultrasonography was normal in cases with a high suspicion of GCA. This culminated in a formal ultrasonography service being commissioned from January 2017. From 2007 to 2009, 162 individuals with suspected GCA were reviewed in NNUH and all of them had biopsies, of which 86 (53\%) were positive. From 2017 to 2019, we saw 419 individuals with suspected GCA, of which 69 (16.5\%) needed a biopsy, of which 20 were positive. The numbers diagnosed on the basis of TAB and ultrasonography are shown in Table 1.

\section{Patient empowerment}

Specialist nurses have a positive impact on patient care and patients themselves hold specialist nurses in high regard. ${ }^{9}$ Individuals with GCA have complex needs and potentially organthreatening disease. Patients with vasculitis who have received education have been found to have better outcomes. ${ }^{10}$ The specialist nurse is ideally placed to provide education to patients about their condition and management. Fear and anxiety can impede a patient's ability to retain and recall information especially when they are not feeling well. ${ }^{11}$ By providing a nurseled outpatient service, patients have the opportunity for regular contact with the vasculitis nurse.

The process of empowering patients started with the establishment of a formal GCA service which ensured that all patients received written information about the disease and the drugs, and a printed regimen tailored to their gender, height and weight. The appointment of a specialist nurse in November 2019 has meant that each patient has time to discuss their management in detail. This holistic approach has enabled patients to benefit from discussions on treatment issues, disease activity and psychosocial aspects. At diagnosis, all patients receive a 30-minute appointment for education about GCA, prednisolone and any further immunosuppression that might be needed, with updates as necessary. Patients also receive an emergency contact number. They can either contact the team directly in the working day or leave a voicemail for urgent support. At every follow-up appointment, patients receive copies of their consultation letters and all correspondence between the GCA service and the primary care physician. All requests for support on aspects of daily living and health involving either GCA or its treatment are handled by the vasculitis nurse. All patients with suspected relapse undergo further ultrasonography to look at the state of the vessels and further immunosuppression is offered as appropriate sequentially with methotrexate and tocilizumab. ${ }^{12}$

\section{Cost-effectiveness of the service}

We have managed to create a service worthy of a national award in the space of 10 years. This has been supported by expansion of staff ( 1 band 7 vasculitis specialist nurse) and the purchase of highend equipment (GE Logiq e ultrasound machine with 4-12 MHz, 8-18 $\mathrm{MHz}$ and $10-22 \mathrm{MHz}$ probes). The capital investment for the ultrasonography machine was supported in its entirety by the Norfolk and Norwich Hospitals Charity (Charity Number 1048170).

To compare the costs of making a diagnosis by ultrasonography \pm biopsy vs first-line biopsy for all, we compared our diagnostic throughput in the years 2007-2009 (prior to any pathway) vs 2017-2019 (full diagnostic pathway in place). For ease of comparison, we used the 2018-2019 agreed tariffs for all the data. TAB costs the NHS, a minimum of $£ 1,284$ per procedure (cost code YQ41B). The cost rises to $£ 1,948$ (cost code YQ41A) in the presence of comorbidities. The cost of a vascular ultrasound scan is $€ 51$ (cost code RD47Z). Table 2 shows the costs of running the service now as compared to the cost of running it 10 years ago (corrected to 2018-2019 tariff). The cost of running the service as it used to run in 2007-2009, without the innovation of ultrasonography would be a minimum of $419 \times £ 1,284=$ $£ 537,996$. In 3 years, we have saved $£ 428,184$ to the UK health economy in GCA diagnostics.

\section{Discussion}

In 2009, problems with the care of individuals with GCA were identified. Over the subsequent 10 years, a series of interconnected projects facilitated the evolution of the GCA service. Referral numbers now seen are commensurate with the published literature. The various projects have improved the patient journey and its timeliness; improved by way of introduction of ultrasonography; harmonised treatments in line with international published standards; empowered patients by educating them and giving 
Table 2. Corrected costs for the diagnostics in our service using cost codes from 2018 to 2019

\begin{tabular}{llllll} 
Years & No. of referrals & Cost of TAB ${ }^{a}$ & Cost of ultrasonography & Total cost & Cost per GCA diagnosis \\
$2007-2009$ & 162 & $£ 208,008$ & $£ 0$ & $£ 208,008$ & $£ 1,284.00$ \\
$2017-2019$ & 419 & $£ 88,596$ & $£ 21,216$ & $£ 109,812$ & $£ 262.08$ \\
\hline
\end{tabular}

$\mathrm{TAB}=$ temporal artery biopsy.

${ }^{\mathrm{a}}$ The costs do not consider the presence of comorbidities.

them access to urgent advice and holistic care; and reduced the burden on primary care by ensuring that glucocorticoid taper and monitoring is looked after by a multidisciplinary team and relapses are diagnosed rapidly and objectively.

Effecting change in the NHS is difficult. To create a service with almost no resources is uniquely exigent. The National Institute of Health and Clinical Excellence measures the cost-benefit of any service or technology based on the concept of quality-adjusted lifeyears. If a change costs up to $€ 30,000$ per quality-adjusted life-year, then it is considered to be cost-effective. Our service has not been formally costed, but the improvements in the quality of care using the metrics of numbers of patients seen, the speed of diagnosis, the education and empowerment of patients, the disease monitoring and quick diagnosis of relapses doubtlessly have resulted in positive change to the quality-adjusted life-years of our cohort of individuals with GCA. Centralising and unifying this patient service has meant that change has been instituted without a concomitant increase in the number of consultant or junior medical staff. The sum total of staffing change has been the addition of a vasculitis specialist nurse. The total capital investment was for an ultrasound machine which costed less than $£ 30,000$ and was covered by charitable funds. As a result of ultrasonography, diagnosis of every case of GCA costs about $£ 1,000$ less than it used to 10 years ago. We are certain that our service would result in a net saving per quality-adjusted life-year improved. This is in line with the experience in South Norway where they showed that their fast-track GCA ultrasonography service saved over 37,000 Norwegian Kroner per patient in all costs in $2013 .^{13}$ In the UK, the development of a fast-track ultrasonography pathway has resulted in quicker diagnosis. ${ }^{14}$ Our work has seen a more holistic improvement in the care of our patients.

We recognise the limitations of our work. It is a single-centre project. It has not been subjected to a formal health economic analysis. It is in the East of England which has more incident GCA than in the rest of the UK. ${ }^{4}$ But we have shown that with multidisciplinary input and teamwork, it is possible to improve the care of a cohort of patients without incurring huge costs. We have published our developments and continue to endeavour to improve the body of knowledge in this area. ${ }^{2,5,6,15}$.

\section{Acknowledgements}

The authors would like to thank Chetan Mukhtyar's trainees Chloe Lapraik, Louise Hamilton, Anita Weerakoon, Khadi Afridi, Max Yates, Eleana Ntatsaki, Mark Sykes, Poonam Sharma, Rasha Briar, Rita Abdulkader, Alice Malpas, Cee Yong, Anoushka Seneviratne, Lauren Steel, Sadaf Saeed, Dimos Merinopoulos, Abid Yousuf and Rifat Mazumder in Rheumatology during this time; Colin Jones's trainees Matt Schneiders and Sofia Habib in Ophthalmology; Chandrashekhara Rai and Anne Howden for supporting the service; Norfolk and Norwich Hospitals Charity for funding the ultrasound machine; Professor Max Bachmann for his advice on costing the service; and Ms Sarah Walker for her role as operational manager for the business cases.

\section{References}

1 Jennette JC, Falk RJ, Bacon PA et al. 2012 revised International Chapel Hill Consensus Conference Nomenclature of Vasculitides. Arthritis Rheum 2013;65:1-11.

2 Mukhtyar $\mathrm{C}$, Hodgson $\mathrm{H}$. The need to establish standards of care for giant cell arteritis. Rheumatology (Oxford) 2020;59:702-4.

3 Mukhtyar C, Guillevin L, Cid MC et al. EULAR recommendations for the management of large vessel vasculitis. Ann Rheum Dis 2009;68:318-23.

4 Smeeth L, Cook C, Hall AJ. Incidence of diagnosed polymyalgia rheumatica and temporal arteritis in the United Kingdom, 19902001. Ann Rheum Dis 2006;65:1093-8.

5 Mukhtyar C, Cate H, Graham C et al. Development of an evidencebased regimen of prednisolone to treat giant cell arteritis - the Norwich regimen. Rheumatol Adv Pract 2019;3:rkz001.

6 Mukhtyar C, Myers H, Scott DGI et al. Validating a diagnostic GCA ultrasonography service against temporal artery biopsy and longterm clinical outcomes. Clin Rheumatol 2020;39:1325-9.

7 Dasgupta B, Borg FA, Hassan N et al. BSR and BHPR guidelines for the management of giant cell arteritis. Rheumatology (Oxford) 2010:49:1594-7.

8 Schmidt WA, Kraft HE, Volker L et al. Colour Doppler sonography to diagnose temporal arteritis. Lancet 1995;345:866.

9 RCN Policy Unit. Specialist nurses make a difference. Policy Briefing 14/2009. London: Royal College of Nursing, 2009:19.

10 Yates M, Watts RA, Bajema IM et al. EULAR/ERA-EDTA recommendations for the management of ANCA-associated vasculitis. Ann Rheum Dis 2016;75:1583-94.

11 Mooney J, Poland F, Spalding $N$ et al. 'In one ear and out the other - it's a lot to take in': a qualitative study exploring the informational needs of patients with ANCA-associated vasculitis. Musculoskeletal Care 2013;11:51-9.

12 Guillevin L, Mukhtyar C, Pagnoux C et al. Conventional and biological immunosuppressants in vasculitis. Best Pract Res Clin Rheumatol 2018;32:94-111.

13 Diamantopoulos AP, Haugeberg G, Lindland A et al. The fast-track ultrasound clinic for early diagnosis of giant cell arteritis significantly reduces permanent visual impairment: towards a more effective strategy to improve clinical outcome in giant cell arteritis? Rheumatology (Oxford) 2016;55:66-70.

14 Patil P, Williams M, Maw WW et al. Fast track pathway reduces sight loss in giant cell arteritis: results of a longitudinal observational cohort study. Clin Exp Rheumatol 2015;33:S-103-6.

15 Mukhtyar $\mathrm{C}$, Myers $\mathrm{H}$, Jones $\mathrm{C}$ et al. The relationship between glycated haemoglobin levels and the risk of giant cell arteritis - a case-control study. Rheumatol Adv Pract 2020;4:rkaa018.

Address for correspondence: Dr Chetan Mukhtyar, Norfolk and Norwich University Hospital, Colney Lane, Norwich NR4 7UY, UK.

Email: chetan.mukhtyar@nnuh.nhs.uk 